Apidologie, 1980, 11 (3), 193-202.

\title{
LA CIRE, SON RECYCLAGE ET SON RÔLE PROBABLE A L'INTERIEUR D'UNE COLONIE D'APIS MELLIFICA
}

\section{Das Wachs, seine Wiederverwendung und seine vermutliche Bedeutung innerhalb eines Volkes von Apis mellifica}

\author{
Roger DARCHEN* \\ Station Biologique, 24620 Les Eyzies
}

\begin{abstract}
RÉSUMÉ
A la suite de marquages radio-actifs de quelques centimètres cubes de cire dans une ruche, il a été possible de connaître l'ampleur et la rapidité de leurs déplacements par les abeilles : il a été ainsi prouvé qu'en quelques heures la cire se trouvait répartie inégalement un peu sur tous les rayons. Grâce à ces expériences, on devine encore que la cire, outre son rôle de matériau de construction, fait sans doute partie du groupe des substances chimiques utiles au maintien de l'interattraction et de la cohésion des individus des sociétés d'abeilles.
\end{abstract}

\section{SUMMARY \\ THE WAX CYCLE IN A BEEHIVE OF APIS MELLIFICA AND ITS VARIOUS FUNCTIONS}

By tagging a few cubic centimeters of wax with radio-active tracers it proved possible to observe the speed and volume of movement of wax in the hive. Within a few hours the marked wax was found unequally distributed in the various combs. From the results, it is suggested that, besides its role of building material, wax may also belong to the group of natural substances which play a part in the interattraction and the maintenance of cohesion among individuals of a bee society

Le cycle de la cire d'Abeille à l'intérieur d'une ruche est assez connu depuis plusieurs années (DARCHEN, 1959, 1972; WENER-MEYER, 1960). On a découvert en particulier que la cire a beau être un produit de la ruche, elle reste tout de même une matière

* Avec l'assistance technique et matérielle du Commissariat à l'Énergie Atomique. Section d'Applications des Radio-éléments (C.E.N. - SACLAY). 
précieuse qui ne se gaspille pas : lorsqu'elle n'est pas utilisée, plus précisément en automne et en hiver, les abeilles la mettent en réserve discrètement sur le pourtour des cellules qui sont alors momentanément, légèrement épaissies. Lorsque le besoin s'en fait sentir, les ouvrières viennent en arracher des débris qu'elles pétrissent et insèrent dans de nouvelles constructions, à savoir de nouveaux rayons ou de nouveaux opercules. La cire est donc régulièrement recyclée à une vitesse qui dépend directement, en grande partie, du cycle biologique de la colonie et, indirectement, du cycle climatique.

Ce phénomène n'est d'ailleurs pas le propre d'Apis mellifica mais de tous les apides sociaux, comme les Trigones, les Mélipones. Chez ces derniers, les mouvements de la cire sont plus importants, surtout parce que les rayons du couvain sont sans cesse totalement remaniés.

Ón savait donc qu'il existait un brassage de la cire dans la ruche, mais on ignorait son ampleur parce que les moyens techniques faisaient encore défaut, ou, du moins, étaient encore peu utilisés dans ce type de recherches.

\section{MÉTHODE EXPÉRIMENTALE}

Les premières expériences n'ont malheureusement pas pu être effectuées avant le début du mois de septembre qui coïncide à la fin de l'année apicole. Les reines pondent peu, les abeilles font des réserves et se préparent à hiverner. Cependant à cette époque là, un certain renouveau du comportement de construction peut se manifester dans la mesure où les abeilles reçoivent un appoint de sucre.

En plein air, deux fortes colonies d'abeilles reçurent chacune $10 \mathrm{~cm}^{3}$ de cire marquée par un produit radioactif différent, du Lipiodol U.F. ou de la Sélénométhionine. Ces formes chimiques ont été choisies car elles sont solubles dans la cire ou miscibles à ce matériau.

TABL. I. - Caractéristiques des traceurs utilisés dans les expériences

TAB. I. - Merkmale der bei diesen Versuchen benu: ten Tracer

\begin{tabular}{l|c|c}
\hline \hline & \multicolumn{1}{|c|}{$\begin{array}{c}\text { Traceur à courte période } \\
\text { Tracer von kurzer Periode }\end{array}$} & $\begin{array}{c}\text { Traceur à longue période } \\
\text { Tracer von langer Periode }\end{array}$ \\
\hline $\begin{array}{l}\text { Radionucléide } \\
\text { Radionukleid }\end{array}$ & Iode ${ }^{131} \mathrm{I}$ & Selenium "'Se \\
\hline $\mathrm{E} \gamma \mathrm{Mev}$ & $0,08-0,72$ & $0,025-0,4$ \\
\hline $\mathrm{T}_{1 / 2}$ & $8,04 \mathrm{j}$ & $120 \mathrm{j}$ \\
\hline $\begin{array}{l}\text { Molécule } \\
\text { Molekül }\end{array}$ & $\begin{array}{l}\text { Esters éthyliques et glycériques } \\
\text { des acides gras } \\
\text { Äthyl- und Glycerinester } \\
\text { von Fettsäuren }\end{array}$ & $\begin{array}{l}\text { Sélénométhionine } \\
\text { Selenmethionin }\end{array}$ \\
\hline $\begin{array}{l}\text { Radio-activité utilisée } \\
\text { Benutzte Radioaktivität }\end{array}$ & $0,1 \mathrm{mCi}$ & $0,1 \mathrm{mCi}$ \\
\hline \hline
\end{tabular}


La cire liquéfiée contenant l'un ou l'autre des traceurs a été répartie, avec un pinceau, sur les bords des cellules du couvain sur une surface d'environ $100 \mathrm{~cm}^{2}$ de chaque côté d'un seul cadre ayant une aire de $1130 \mathrm{~cm}^{2}$. Le choix de cette partie du rayon se justifiait par la date tardive d'expérimentation. Les cellules autour du couvain étaient encore l'objet de soins très attentifs. Le rayon ainsi marqué a été accroché dans la ruche à la place du troisième cadre à partir de la paroi verticale du corps de ruche.

Après le marquage, une rapide détection à l'aide d'une sonde portative (type SPP 2) a permis de localiser rapidement les zones radio-actives. Celles-ci ont été ensuite cartographiées au laboratoire à l'aide d'une chaine de mesure comprenant une sonde à scintillation dont le cristal a les dimensions suivantes : diamètre $38 \mathrm{~mm}$, hauteur $25 \mathrm{~mm}$. Cette sonde est reliée à une échelle de comptage ECP 522 . La sonde, insérée verticalement dans un bloc de plomb mobile sur des rails, se trouve à $5 \mathrm{~cm}$ au-dessus du rayon de cire, lui-même placé horizontalement sur un chariot pouvant être déplacé de gauche à droite et d'avant en arrière (cf. Courtois, Lecomte, 1958; Courtois, Lecomte, Salleron, 1961; Douault, Roger, AZcuF, 1973). On a pu ainsi déterminer 32 points de mesure sur un cadre standard Dadant. A chaque fois, nous avons effectué 10 comptages de 30 secondes qui nous ont permis de procéder à quelques calculs statistiques.

Les deux ruches d'expériences du type Dadant, contenaient 10 cadres, et étaient surmontées d'une hausse. Cette dernière était équipée de 9 cadres qui, au moment de ce travail, ne contenaient ni provisions, ni couvain.

Les abeilles étaient toutes de la race Apis mellifica mellifica.

\section{RÉSULTATS}

\section{Première série d'expériences effectuées avec l'iode 131 (lipiodol U.F.)}

Le compteur portatif nous a permis de découvrir que tous les cadres de la ruche et de la hausse devenaient radio-actifs dans les 24 heures qui suivaient l'introduction du traceur. L'examen de chaque cadre isolé en dehors de la ruche nous a révélé en outre que la radio-activité décroissait en fonction de l'éloignement du cadre marqué. La cire des hausses, sauf celle du cadre situé au-dessus du cadre marqué, était la moins radioactive.

Trois jours après le début de cette expérience, j'ai retiré le cadre construit, voisin de celui qui nous avait servi à introduire l'iode 131. Je l'ai remplacé par un support horizontal en bois accroché à la crémaillère soutenant les cadres. Une languette de cire gaufrée de $56 \mathrm{~mm}$ de large et $60 \mathrm{~mm}$ de long y était soudée verticalement. La cire fut alors immédiatement étirée et très rapidement les dimensions du nouveau rayon dépassèrent celles de la languette. Tout le rayon devint radio-actif mais les cellules les plus profondes et celles de l'extrémité du rayon l'étaient davantage, prouvant ainsi que les abeilles constructrices ne s'étaient pas contentées d'utiliser la cire de la languette pour édifier leur nouveau rayon mais qu'elles étaient allées chercher des matériaux sur les rayons voisins (Fig. 1 et 2 , autoradiographie du rayon en question).

Cette expérience nous a donc apporté des données intéressantes et nouvelles, 1) sur la rapidité du déplacement de la cire à travers la ruche, 2) sur l'ampleur de ses déplacements puisque la cire se retrouve dans tous les recoins de l'habitation, 3) sur le mélange de la cire fournie par l'apiculteur et celle qui est récupérée par les abeilles lors 


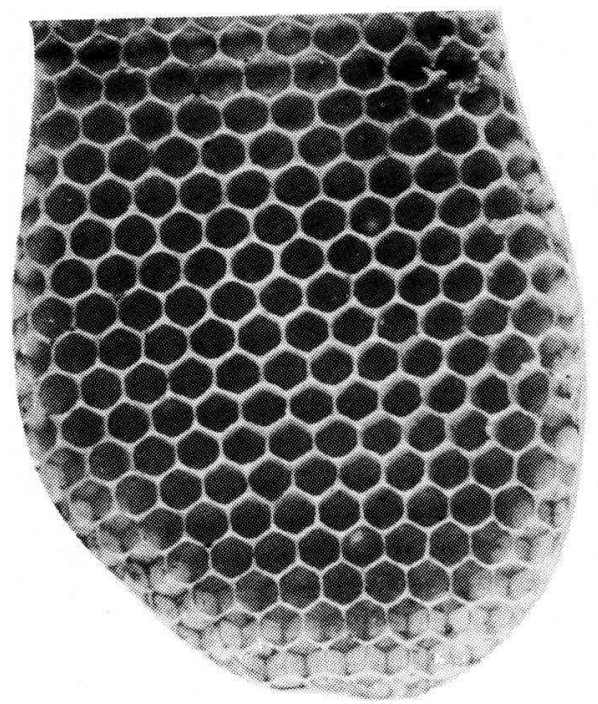

FIG. 1. - Rayon construit sur un morceau de cire gaufrée.

Авв. 1. - Wabe ausgebaut auf einem Stück Mittelwand.

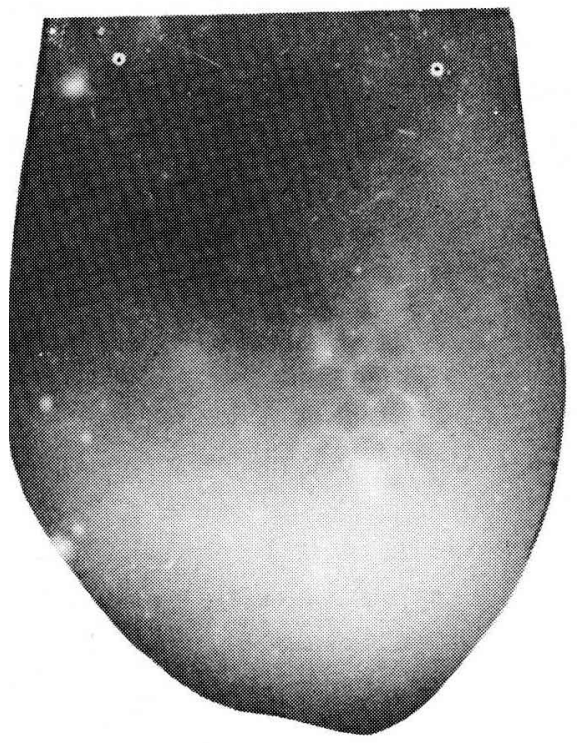

FIG. 2. - Autoradiographie du rayon construit sur le morceau de cire gaufrée. AвB. 2. - Autoradiographie der Wabe die auf einem Stück Mittelwand ausgebaut wurde. 
de l'utilisation de la cire gaufrée, 4) sur la répartition irrégulière de la cire radio-active récupérée sur les différents rayons de la ruche.

Deuxième série d'expériences effectuées avec le selenium 75 (Selenométhionine).

Les résultats ont été exactement les mêmes, mais, grâce à la période beaucoup plus longue de cet élément, nous avons pu effectuer des mesures sur l'un des cadres qui se trouvait séparé par 2 cadres du rayon indiqué, trois mois après le début des expériences (Fig. 3). Elles vont nous permettre de connaitre la répartition irrégulière de la radioactivité sur un seul rayon et d'essayer de la comprendre en fonction des besoins de la colonie.

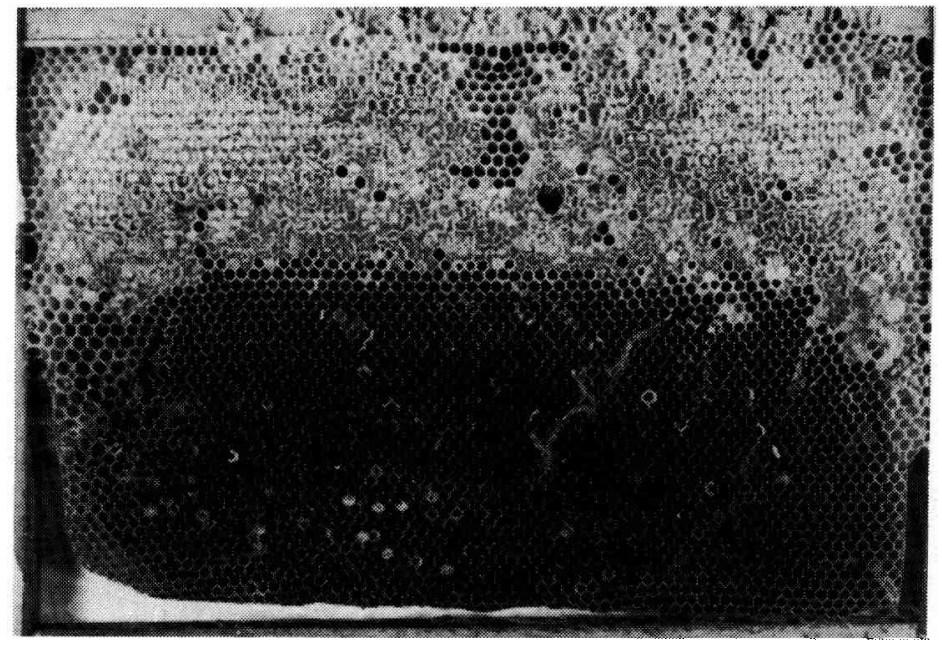

FIG. 3. - Rayon construit dans un cadre Dadall.

La radio-activité de la cire est répartie irrégulièrement (cf. le tableau III).

ABB. 3. - Wabe in einem DADANT-Rähmche'n.

Die Radioaktivität des Wachses ist unregelmässig verteilt (sichc Tab. III).

En comparant les moyennes et les variances les unes avec les autres, j'ai déterminé 10 degrés de radio-activité, 1 correspondant à la plus faible et 10 à la plus forte. Le tableau 3 ci-joint rend compte de leurs répartitions sur le rayon étudié. De plus, comme nous possédons la photographie de ce dernier (Fig. 3) il nous est loisible d'interpréter nos résultats.

Dans un rayon construit d'une ruche, les abeilles ne répartissent pas au hasard leurs réserves et leur élevage : le miel operculé se trouve dans les cellules du haut (ici, il occupe presque la moitié supérieure du rayon), le pollen occupe les cellules immédiate- 


\begin{tabular}{|c|c|c|c|c|c|}
\hline & 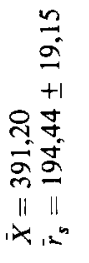 & 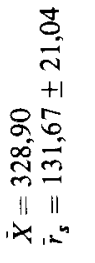 & 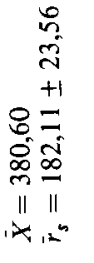 & 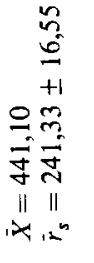 & 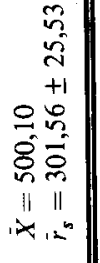 \\
\hline 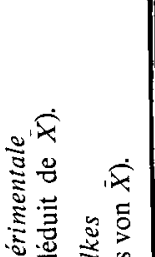 & 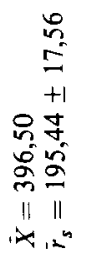 & 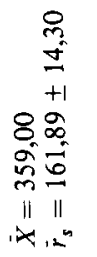 & 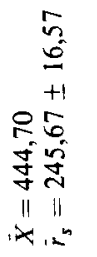 & 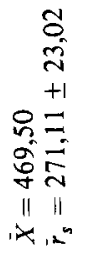 & 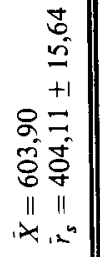 \\
\hline 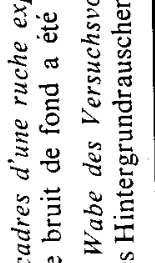 & 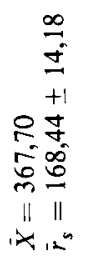 & 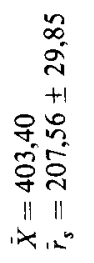 & 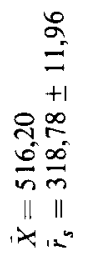 & 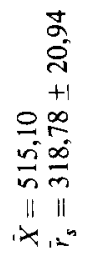 & 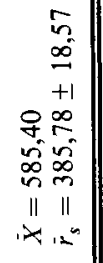 \\
\hline 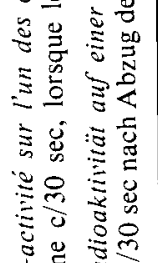 & 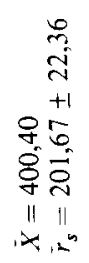 & 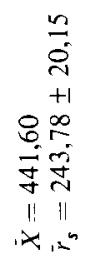 & 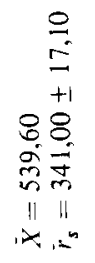 & 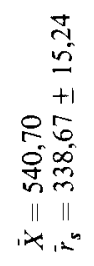 & 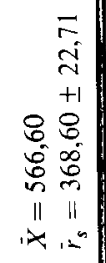 \\
\hline 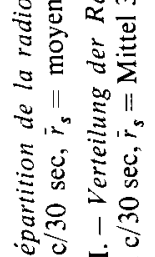 & 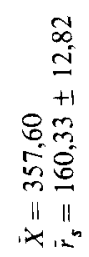 & 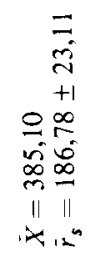 & 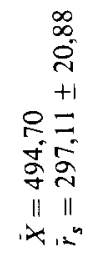 & 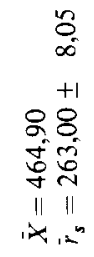 & 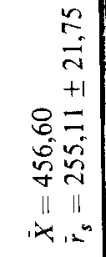 \\
\hline 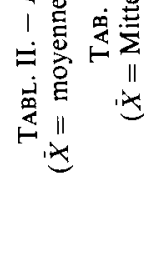 & 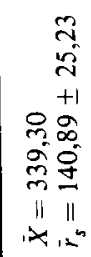 & 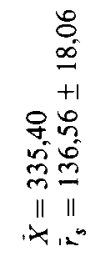 & 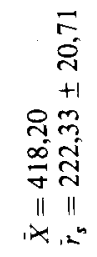 & 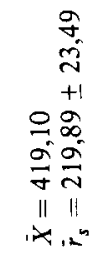 & 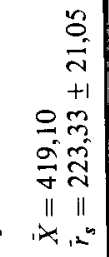 \\
\hline & 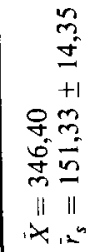 & 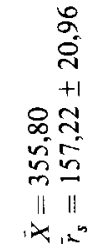 & 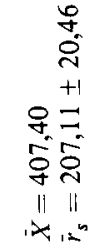 & 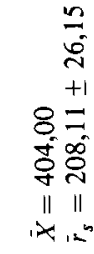 & 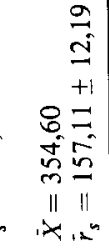 \\
\hline
\end{tabular}


TABL. III. - Répartition inégale de la radio-activité sur un cadre de corps de ruche (le taux de radio-activité va croissant de 1 à 10).

TAB. III. - Unregelmässige Verteilung der Radioaktivität auf einer Wabe aus dem Brutraum (die Radioaktivität steigt von Stufe 1 zu Stufe 10 an).

\begin{tabular}{c|c|c|c|c|c|c}
\hline \hline 1 & 1 & 1 & 3 & 2 & 2 & 2 \\
\hline 1 & 1 & 1 & 6 & 4 & 1 & 1 \\
\hline 3 & 3 & 7 & 9 & 8 & 6 & 2 \\
\hline 3 & 3 & 5 & 9 & 8 & 6 & 6 \\
\hline 1 & 3 & 5 & 9 & 10 & 10 & 6 \\
\hline \hline
\end{tabular}

ment au-dessous et les côtés du rayon, le couvain plus ou moins développé, la partie centrale. La radio-activité est donc faible sur le miel operculé. A cela rien d'étonnant, car lors du début des expériences, les réserves de la ruche étaient constituées et les cellules étaient déjà fermées. Il n'y a donc eu presqu'aucune modification de la plupart d'entre elles. Les opercules seront ouverts durant l'hiver afin de permettre à la colonie de survivre grâce à la consommation du miel.

Les cellules centrales sont les plus radio-actives ainsi que celles de l'extrémité droite du rayon. Puisque notre expérimentation a eu lieu très tardivement dans l'année, la plus grande partie des cellules du centre sont plus ou moins partiellement remplies de miel et le couvain y est absent. Le miel, operculé ou non, occupe donc environ les deux tiers de ce rayon. La radio-activité de la zone centrale et celle des extrémités s'explique donc simplement par la mise en réserve de la cire de récupération sur les bords des cellules, en attendant leur réutilisation au printemps suivant. Il est intéressant d'ailleurs de constater que, dans les parties les plus radio-actives $(9,10)$, les bourrelets de cire des orifices des cellules sont beaucoup plus volumineux que partout ailleurs.

\section{DISCUSSION}

Cette radio-activité généralisée et rapide de toute la ruche peut-elle être attribuée à une contamination par les pattes des insectes? On ne peut douter que les abeilles y contribuent un peu : on sait en effet que, dans ce type d'expériences, on n'élimine jamais totalement cette possibilité et qu'on est obligé d'en tenir plus ou moins compte (Douault, 1966). Cependant, ici, il ne fait aucun doute qu'elle ne peut à elle seule rendre compte des résultats que nous avons observés. En effet, $r$. l'avons vu, l'irrégularité de la répartition de la radio-activité sur les rayons est pa. ticulièrement liée au rôle dévolu à chaque partie de la bâtisse, à savoir, rappelons-le, le centre pour l'élevage du couvain, le sommet pour la mise en réserve du miel, les autres cellules pour l'emmagasinage provisoire de la cire. Dans notre cas particulier, les cellules du couvain où les 
abeilles travaillèrent le plus et les cellules qui servirent à entasser la cire supplémentare à la fin de l'été se montrèrent les plus radio-actives. En revanche, les cellules définitivement operculées ne furent l'objet que de peu de soins et devinrent peu radio-actives. Enfin, l'autoradiographie (Fig. 2) à elle seule élimine les derniers doutes concernant l'importance possible de la contamination de la cire par les abeilles. On sait en effet que, lors des constructions sur de la cire gaufrée, les abeilles commencent à utiliser la cire qui leur est fournie : en grattant la feuille gaufrée qui porte sur elle, gravée la forme des fonds de cellules, les ouvrières prélèvent des miettes de cire leur permettant d'élever, au moins partiellement, les parois des cellules. On comprend alors que cette zone de rayon en construction soit beaucoup moins radio-active puisque les constructrices se contentent d'utiliser la cire neuve et neutre mise à leur disposition. En revanche, lorsqu'elles développent des édifices au-delà des limites de la cire gaufrée qui leur était initialement offerte, elles sont obligées de sécréter de la cire ou d'aller récupérer du matériau dans les diverses parties de la ruche, en l'occurence celui qui se trouvait tout à côté et sur lequel on avait déposé de la cire marquée à l'I 131 .

Ce travail confirme donc les observations antérieures que nous avons rappelées. Il nous démontre ensuite l'ampleur du brassage, rapide et continuel de la cire à travers toute la ruche (hausse comprise) : aucun recoin de l'habitation ne peut être exempt d'une contamination radio-active car, à un moment ou a un autre de la vie de la colonie, il peut être soumis à une transformation nécessaire au besoin de la population. On devine cependant déjà qu'il y a des zones où les mouvements de la cire sont plus fréquents; ils correspondent à celles qui sont en relation avec le développement du couvain et des réserves ou bien à celle où s'édifient de nouvelles cellules. Il semble malgré tout que certaines parties des bâtisses peu utilisées en général en pleine saison apicole, dans le bas et le pourtour inférieur des rayons, deviennent des lieux privilégiés de mise en réserve de la cire en automne.

Ces mouvements de la cire sont à rapprocher de ceux du miel ou du nectar qui nous ont été signalés précédemment (Douault, Louveaux, TheurkaufF, Azceuf, Pintena, 1974; Douault, Roger, Pain, 1975; Kloft, Djalal, Drescher, 1976). Ils ont en commun la manifestation de remaniements plus ou moins importants, fréquents, rapides, perpétuels et irréguliers à l'intérieur de la ruche. Une colonie dont quelques individus seulement ont été marqués avec de l'or 198 devient totalement radioactive en 36 heures.

Enfin, tous ces transferts de la cire n'évoquent-ils pas ceux des nombreuses phéromones des abeilles? Ils en ont toutes les caractéristiques. Tout en n'étant pas une phéromone sensu stricto, la cire pourrait avoir une action semblable du fait même de sa composition ou du fait qu'elle est le support de parfums et de phéromones d'interattraction ou de familiarisation : tous les éleveurs de reines savent par exemple très bien qu'ils doivent déposer dans les ruches pendant quelques heures les cupules de cire qui leur serviront ensuite à déposer de jeunes larves, s'ils veulent que leur acceptation par les ouvrières soit satisfaisante. De même, récemment LeNSKY et SLABEZKI (1978), ont démontré 
que le dépôt de la sécrétion huileuse des tarses de la reine sur les rayons et de celle de ses glandes mandibulaires entrainait une inhibition de la construction de cellules royales. La cire semble donc prendre désormais une autre dimension dans la colonie : elle était, et est encore, le matériau de construction qui forme le squelette de la ruche, le tissu d'emmagasinage des réserves et d'élevage du couvain, elle devient en outre, pour nous, maintenant un des liants favorisant la cohésion de la société.

Reçu pour publication en mai 1980. Eingegangen im Mai 1980.

\section{ZUSAMMENFASSUNG}

Der Zyklus des Wachses innerhalb eines Bienenstockes ist seit einigen Jahren teilweise bekannt. In dieser Arbeit haben wir versucht, seine Bewegungen und das Ausmass seiner Verlagerung mit Hilfe von zwei radioaktiven Markern, Jod 131 und Selen 75, noch feiner zu analysieren. Als Ergebnis unserer Versuche haben wir die folgenden Hauptfeststellungen getroffen :

1. Die Verlagerung des Wachses, das auf den Zellen einer Wabe deponiert worden worden war, erfolgte rasch.

2. Das Ausmass dieser Verlagerung ist beträchtlich, da schon 24 Stunden nach der Einbringung der Marker alle Waben des Brutraumes und sogar des Honigraumes kontaminiert sein können.

3. Die Verteilung des radioaktiven Stoffes auf die Gesamtheit der Waben ist sehr unregelmässig. Diese Unregelmässigkeit steht in Beziehung zur funktionellen Rolle jedes Abschnittes des Bauwerks und zum biologischen Rhythmus des Bienenvolkes zu einem gegebenen Augenblick im Jahresablauf : Zur Zeit unserer Versuche im Monat September, der schon ein wenig eine Periode der Vorbereitung zur Einwinterung ist, begannen die Bienen ihr Wachs als Vorrat an den Zellrändern an den unteren Wabenabschnitten abzulagern.

Diese beträchtlichen und raschen Transporte von Wachs erinnern an diejenigen der zahireichen Pheromone der Bienen. Sie zeigen alle Charakteristika von diesen. Obwohl es sich um kein Pheromon im engeren Sinne handelt, könnte das Wachs doch eine ähnliche Funktion haben. Und zwar sowohl aufgrund seiner Zusammensetzung wie auch als Träger von Gerüchen und Pheromonen der gegenseitigen Anziehung und der Zusammengehörigkeit.

\section{BIBLIOGRAPHIE}

Courtois G., Lecomte J., 1958. - Sur un procédé de marquage des abeilles butineuses au moyen d'un radio-isotope. C. $R$. Acad. Sci., 247, 147-149.

Courtois G., Lecomte J., Salleron F., 1961. - Étude des échanges de nourriture à l'intérieur de la ruche entre les abeilles ouvrières (Apis mellifica L.). C. R. Acad. Sci., 252, 1057-1059.

DarChen R., 1959. - Les techniques de construction chez Apis mellifica L., Ann. Sci. Nat., 12, 113-209.

DARCHEN R., 1972. - Étude comparative de l'économie des matériaux de construction chez divers Apides Sociaux, Apis, Trigona et Melipona (Apidés). Rev. Comp. Animal, 6, 201-215.

Douault Ph., 1966. - Étude de la contamination externe de l'abeille Apis mellifica et son milieu par un radio-isotope introduit dans la nourriture. Ann. Abeille, 9, 37-45.

Douault Ph., Roger B., Azcuf P., 1973. - Étude, au moyen d'un radio-isotope de deux transferts successifs de nourriture entre ouvrières d'abeilles (Apis mellifica ligustica Spinola). C. R. Acad. Sci., Paris, 277, 333-336. 
Douault Ph., Louveaux J., Theurkauff J., Azeuf P., Pintena J., 1974. - Étude du stockage des réserves par les abeilles au moyen d'un sirop de sucre marqué au 141 Ce. Apidologie, 5, 2, 107-126.

Douault Ph., Roger B., Pain J., 1975. - Étude comparative, au moyen d un radio-isotope des transferts de nourriture entre les abeilles des trois castes d'Apis mellifica ligustica Spin. C. R. $25^{\mathrm{e}}$ Congrès Apic., Apimondia, Grenoble, 307-308.

LENSKY Y., SLABEZKI Y., 1978. - The inhibiting effect of the queen bee (Apis mellifica L.) foot-print pheromone on the construction of swarming queen cups. C. R. Triwak Bee Rescarch Center, 1-31.

WENER-MeYer W., 1960. - Wachs und Wachsban Kittharz. In Biene und Bienchzuchll, 202-232 Ehren wirth Edit.

KLoft W. J., Djalal A. S., Drescher W., 1976. - Untersuchung der unterschiedlichen Futterverteilung in Arbeiterinnensgruppen verschiedener Rassen von Apis mellifica L. mit Hilfe von $32 \mathrm{P}$ als Tracer. Apidologie, 7, 1, 49-60. 